\title{
The Maxwell Electromagnetic Equations and the Lorentz Type Force Derivation-The Feynman Approach Legacy
}

\author{
Anatoliy K. Prykarpatsky • Nikolai N. Bogolubov Jr.
}

Received: 5 April 2011 / Accepted: 15 July 2011 / Published online: 12 August 2011

(C) The Author(s) 2011. This article is published with open access at Springerlink.com

\begin{abstract}
R. Feynman's “heretical” approach (Dyson in Am. J. Phys. 58:209-211, 1990; Dyson in Phys. Today 42(2):32-38, 1989) to deriving the Lorentz force based Maxwell electromagnetic equations is revisited, the its complete legacy is argued both by means of the geometric considerations and its deep relation with the vacuum field theory approach devised (Prykarpatsky et al. in Int. J. Theor. Phys. 49:798-820, 2010; Prykarpatsky et al. in Preprint ICTP, 2008, http://publications.ictp.it). Being completely classical, we reanalyze the Feynman's derivation from the classical Lagrangian and Hamiltonian points of view and construct its nontrivial relativistic generalization compatible with the vacuum field theory approach.
\end{abstract}

Keywords Feynman's approach · Lorentz force · Relativistic electrodynamics · Least action principle $\cdot$ Lagrangian and Hamiltonian analysis

"A physicist needs that his equations should be mathematically sound and that in working with his equations he should not neglect quantities unless they are small"

(P.A. M. Dirac)

\footnotetext{
A.K. Prykarpatsky $(\bowtie)$

Department of Mining Geodesy, AGH University of Science and Technology, Krakow 30059, Poland e-mail: pryk.anat@ua.fm
}

\author{
A.K. Prykarpatsky \\ Ivan Franko Pedagogical State University, Drohobych, Lviv region, Ukraine \\ e-mail: prykanat@cybergal.com \\ N.N. Bogolubov Jr. \\ V.A. Steklov Mathematical Institute of RAS, Moscow, Russian Federation \\ e-mail: nikolai_bogolubov@hotmail.com \\ N.N. Bogolubov Jr. \\ The Abdus Salam International Centre of Theoretical Physics, Trieste, Italy
}




\section{Introduction}

In 1948 R. Feynman presented but did not published [1,2] a very interesting, in some aspects "heretical", quantum-mechanical derivation of the classical Lorentz force acting on a charged particle under the influence of an external electromagnetic field. His result was analyzed by many authors $[3-9,11,24]$ from different points of view, including its relativistic generalization [10]. As this problem is completely classical, we reanalyze the Feynman's derivation from the classical Hamiltonian dynamics point of view on the coadjoint space $T^{*}(N), N \subset \mathbb{R}^{3}$, and construct its nontrivial generalization compatible with results $[15,16]$ of Sect. 1, based on a recently devised vacuum field theory approach [15, 18]. Having further obtained the classical Maxwell electromagnetic equations we supply the complete legacy of Feynman's approach to the Lorentz force and demonstrate its compatibility with the relativistic generalization presented in $[15-18,20]$.

Consider the motion of a point particle with a charge $\xi \in \mathbb{R}$ and located at point $q \in \mathbb{R}^{3}$ on time $t \in \mathbb{R}$, which is under the influence of an external smooth vector magnetic potential $A \in C^{\infty}\left(\mathbb{R} \times \mathbb{R}^{3} ; \mathbb{R}^{3}\right)$ measured, respectively, subject to the reference frame $\mathcal{K}(t, q)$. For its geometrical description, following [14, 21, 22], it is convenient to introduce a trivial fiber bundle structure $\pi: M \rightarrow N, M=N \times G, N \subset \mathbb{R}^{3}$, with the Abelian structure group $G:=$ $\mathbb{R} \backslash\{0\}$, equivariantly acting [12] on the canonically Poissonian coadjoint space $T^{*}(M)$, and to endow it with a general connection one-form $\mathcal{A}: M \rightarrow T^{*}(M) \times \mathcal{G}$ as

$$
\mathcal{A}(q ; g):=\langle\mathcal{A}(q, g), \xi\rangle_{\mathcal{G}}+g^{-1} d g
$$

on the phase space $M$, where $q \in N$ and $g \in G$. If $l: T^{*}(M) \rightarrow \mathcal{G}^{*}$ is the related momentum mapping, one can construct [12] the reduced phase space $\overline{\mathcal{M}}_{\xi}:=l^{-1}(\xi) / G \simeq T^{*}(N)$, where $\xi \in G \simeq \mathbb{R}$, is taken to be fixed, possessing the reduced canonical symplectic structure

$$
\omega_{\xi}^{(2)}(q, p)=\langle d p, \wedge d q\rangle+\xi d\langle A(q), d q\rangle
$$

on $T^{*}(N)$, where we put, by definition, that $\mathcal{A}(q ; g):=\langle A(q), d q\rangle \in T_{q}^{*}(N)$. From (1.2) one finds easily the respectively reduced Poisson brackets on $T^{*}(N)$ :

$$
\left\{q^{i}, q^{j}\right\}_{\omega_{\xi}^{(2)}}=0, \quad\left\{p_{j}, q^{i}\right\}_{\omega_{\xi}^{(2)}}=\delta_{j}^{i}, \quad\left\{p_{i}, p_{j}\right\}_{\omega_{\xi}^{(2)}}=\xi F_{j i}(q),
$$

where for $i, j=\overline{1,3}$

$$
F_{i j}(q):=\partial A_{j} / \partial q^{i}-\partial A_{i} / \partial q^{j}
$$

is the so-called reduced electromagnetic tensor with respect to the reference frame $\mathcal{K}(t, q)$. If now to introduce a new momentum variable $\tilde{p}:=p+\xi A(q)$ on $T^{*}(N) \ni(q, p)$, it is easy to verify that $\omega_{\xi}^{(2)} \rightarrow \tilde{\omega}_{\xi}^{(2)}:=\langle d \tilde{p}, \wedge d q\rangle$, giving naturally rise to the following "minimal coupling" canonical Poisson brackets [13, 21, 22]:

$$
\left\{q^{i}, q^{j}\right\}_{\tilde{\omega}_{\xi}^{(2)}}=0, \quad\left\{\tilde{p}_{j}, q^{i}\right\}_{\tilde{\omega}_{\xi}^{(2)}}=\delta_{j}^{i}, \quad\left\{\tilde{p}_{i}, \tilde{p}_{j}\right\}_{\tilde{\omega}_{\xi}^{(2)}}=0
$$

for $i, j=\overline{1,3}$ with respect to the "shifted" reference frame $\mathcal{K}_{f}\left(t, q-q_{f}\right)$, characterized by the phase space coordinates $(q, \tilde{p}) \in T^{*}(N)$, iff the corresponding not dynamical Maxwell field equations

$$
\partial F_{i j} / \partial q_{k}+\partial F_{j k} / \partial q_{i}+\partial F_{k i} / \partial q_{j}=0
$$

for all $i, j, k=\overline{1,3}$ with curvature tensor (1.4) are satisfied on $N$. 
This result means, in particular, that the corresponding dynamical system related with a charged particle, which is considered with respect to the reference frame $\mathcal{K}(t, q)$ and under the influence of the smooth external electromagnetic field $A \in C^{2}\left(\mathbb{R} \times \mathbb{R}^{3} ; \mathbb{R}^{3}\right)$, can be equivalently treated as the one with respect to canonical Poisson bracket (1.5) in the "shifted" reference frame $\mathcal{K}_{f}\left(t, q-q_{f}\right)$. The latter property appears to be important for analyzing the related dynamics of a charged particle $\xi$ subject to another charged particle $\xi_{f}$, moving with a velocity $u_{f}$ with respect to the reference frame $\mathcal{K}(t, q)$.

\section{The Lorentz Type Force and Maxwell Electromagnetic Field Equations-The Lagrangian Analysis}

The Poisson structure (1.5) makes it possible to describe a charged particle $\xi \in \mathbb{R}$, located at point $q \in N \subset \mathbb{R}^{3}$, moving with a velocity $q^{\prime}:=u \in T_{q}(N)$ with respect to the reference frame $\mathcal{K}(t, q)$, being under the electromagnetic influence of an external charged particle $\xi_{f} \in \mathbb{R}$ located at point $q_{f} \in N \subset \mathbb{R}^{3}$ and moving with respect to the same reference frame $\mathcal{K}(t, q)$ with a velocity $q_{f}^{\prime}:=u_{f} \in T_{q_{f}}(N)$, where, by definition, $\frac{d}{d t}(\ldots):=(\ldots)^{\prime}$ is the temporal derivative with respect to the temporal parameter $t \in \mathbb{R}$. Really, consider a new reference frame $\mathcal{K}_{f}\left(t, q-q_{f}\right)$ moving with respect to the reference frame $\mathcal{K}(t, q)$ with the velocity $u_{f}$. With respect to the reference frame $\mathcal{K}_{f}\left(t, q-q_{f}\right)$ this point particle with charge $\xi \in \mathbb{R}$ moves with the velocity $u-u_{f} \in T_{q-q_{f}}(N)$ and, respectively, another point particle with charge $\xi_{f} \in \mathbb{R}$ stays in rest. Then one can write down the standard classical Lagrangian function of the charged particle $\xi$ with a constant mass $m \in \mathbb{R}_{+}$subject to the "shifted" reference frame $\mathcal{K}_{f}\left(t, q-q_{f}\right)$ :

$$
\mathcal{L}_{f}\left(q, q^{\prime}\right)=\frac{m}{2}\left|q^{\prime}-q_{f}^{\prime}\right|^{2}-\xi \varphi
$$

and the scalar potential $\varphi \in C^{2}(N ; \mathbb{R})$ is the corresponding potential energy. On the other hand, owing to (2.1) and the Poisson bracket relationships (1.5) the following equality for the canonical momentum of the charged particle $\xi$ with respect to the reference frame $\mathcal{K}_{f}(t, q-$ $q_{f}$ ) holds:

$$
\tilde{p}:=p+\xi A(q)=\delta \mathcal{L}_{f}\left(q, q^{\prime}\right) / \delta q^{\prime}
$$

or, equivalently,

$$
p+\xi A(q)=m\left(q^{\prime}-q_{f}^{\prime}\right),
$$

expressed in the light speed $c=1$ units. Taking into account that the charged particle $\xi$ momentum with respect to the reference frame $\mathcal{K}(t, q)$ equals $p:=m u \in T_{q}^{*}(N)$, one can easily obtain from (2.3) the important relationship

$$
\xi A(q)=-m u_{f}
$$

for the magnetic vector potential $A \in C^{2}\left(N ; \mathbb{R}^{3}\right)$, which was before obtained in $[17,18,23]$ within a recently devised vacuum field theory approach. Taking now into account (2.1) and (2.4) one finds the following Lagrangian equations:

$$
\frac{d}{d t}[p+\xi A(q)]=\partial \mathcal{L}_{f}\left(q, q^{\prime}\right) / \partial q=-\xi \nabla \varphi,
$$


giving rise to the following charged particle $\xi$ dynamics:

$$
\begin{aligned}
d p / d t & =-\xi \partial A / \partial t-\xi \nabla \varphi-\xi\langle u, \nabla\rangle A \\
& =-\xi \partial A / \partial t-\xi \nabla \varphi-\xi\langle u, \nabla\rangle A+\xi \nabla\langle u, A\rangle-\xi \nabla\langle u, A\rangle \\
& =-\xi(\partial A / \partial t+\nabla \varphi)+\xi u \times(\nabla \times A)-\xi \nabla\langle u, A\rangle .
\end{aligned}
$$

As a result of (2.6) we obtain the modified Lorentz type force

$$
d p / d t=\xi E+\xi u \times B-\xi \nabla\langle u, A\rangle,
$$

which slightly differs from the classical Lorentz force expression

$$
d p / d t=\xi E+\xi u \times B
$$

by the gradient term

$$
F_{c}:=-\nabla\langle u, A\rangle,
$$

and which was obtained in $[17,18]$ and simultaneously in [19]. Here we put, by definition,

$$
E:=-\partial A / \partial t-\nabla \varphi, \quad B:=\nabla \times A,
$$

Note that the Lorentz type force expression (2.7) can be naturally generalized to the relativistic case by taking into account that the Lorenz condition

$$
\partial \varphi / \partial t+\langle\nabla, A\rangle=0
$$

is imposed on the electromagnetic potential $(\varphi, A) \in C^{2}\left(N ; \mathbb{R} \times \mathbb{R}^{3}\right)$.

Indeed, from (2.10) one obtains the Lorentz invariant field equation

$$
\partial^{2} \varphi / \partial t^{2}-\Delta \varphi=\rho_{f},
$$

where $\Delta:=\langle\nabla, \nabla\rangle$ and $\rho_{f}: N \rightarrow \mathcal{D}^{\prime}(N)$ is the generalized density function of the external charge distribution $\xi_{f}$. Following now by the calculations from $[17,18]$ we can easily find from (2.12) and the charge conservation law

$$
\partial \rho_{f} / \partial t+\left\langle\nabla, J_{f}\right\rangle=0
$$

the next Lorentz invariant equation on the magnetic vector potential $A \in C^{2}\left(N ; \mathbb{R}^{3}\right)$ :

$$
\partial^{2} A / \partial t^{2}-\Delta A=J_{f}
$$

Moreover, relationships (2.10), (2.12) and (2.14) easily yield the true classical Maxwell equations

$$
\begin{aligned}
& \nabla \times E=-\partial B / \partial t, \quad \nabla \times B=\partial E / \partial t+J_{f}, \\
& \langle\nabla, E\rangle=\rho_{f}, \quad\langle\nabla, B\rangle=0
\end{aligned}
$$

on the electromagnetic field $(E, B) \in C^{2}\left(N ; \mathbb{R}^{3} \times \mathbb{R}^{3}\right)$. 
Consider now the Lorenz condition (2.11) and observe that it is equivalent to the following local conservation law:

$$
\frac{d}{d t} \int_{\Omega_{t}} \varphi d^{3} q=0,
$$

giving rise to the important relationship for the magnetic potential $A \in C^{2}\left(N ; \mathbb{R}^{3}\right)$

$$
A=q_{f}^{\prime} \varphi
$$

with respect to the reference frame $\mathcal{K}(t, q)$, where $\Omega_{t} \subset N$ is any open domain with the smooth boundary $\partial \Omega_{t}$, moving jointly with the charge distribution $\xi_{f}$ in the region $N \subset \mathbb{R}^{3}$ with the corresponding velocity $q_{f}^{\prime}$. Taking into account relationship (2.4) one can easily find the expression for our charged particle $\xi$ 'inertial' mass:

$$
m=-\bar{W}, \quad \bar{W}:=\xi \varphi,
$$

coinciding with that in $[17,18,23]$, where we denoted by $\bar{W} \in C^{2}(N ; \mathbb{R})$ the corresponding potential energy of the charged particle $\xi$ with respect to the reference frame $\mathcal{K}(t, q)$.

\section{The Modified Least Action Principle and the Hamiltonian Analysis}

\subsection{A Moving Charged Point Particle Lagrangian Analysis}

Based on the representations (2.17) and (2.18) one can rewrite the determining Lagrangian equation (2.5) as follows:

$$
\frac{d}{d t}\left[-\bar{W}\left(u-u_{f}\right)\right]=-\nabla \bar{W}
$$

which is completely equivalent to the Lorentz type force expression (2.7) calculated with respect to the reference frame $\mathcal{K}(t, q)$. Indeed, making use of (2.4), the left hand side of relationship (3.1) can be identically rewritten as

$$
\begin{aligned}
\frac{d}{d t}\left[-\bar{W}\left(u-u_{f}\right)\right] & =\frac{d}{d t}(-\bar{W} u)+\frac{d}{d t}(\xi A) \\
& =\frac{d p}{d t}+\xi \frac{\partial A}{\partial t}+\xi\langle u, \nabla\rangle A .
\end{aligned}
$$

Taking into account the condition (2.18) and the identity

$$
\nabla\langle a, b\rangle=\langle a, \nabla\rangle b+\langle b, \nabla\rangle a+b \times(\nabla \times a)+a \times(\nabla \times b),
$$

holding for any smooth vector-functions $a, b \in C^{2}\left(N ; \mathbb{R}^{3}\right)$, we derive from (3.2)

$$
\begin{aligned}
d p / d t & =\xi\left(-\nabla \varphi-\frac{\partial A}{\partial t}\right)+\xi u \times u \times(\nabla \times A)-\xi \nabla\langle u, A\rangle \\
& =\xi E+\xi u \times B-\xi \nabla\langle u, A\rangle,
\end{aligned}
$$

that is exactly the Lorentz type force (2.7), obtained above. 
Remark 3.1 It is interesting to remark here that (3.1) does not allow the Lagrangian representation with respect to the reference frame $\mathcal{K}(t, q)$ in contrast to that of (2.5).

The remark above is a challenging source of our further analysis concerning the relativistic generalization of the Lorentz type force (2.7). Namely, the following proposition holds.

Proposition 3.2 The Lorentz type force (2.7), in the case where the charged particle $\xi$ momentum is defined owing to (2.18) as $p=-\bar{W} u$, is the exact relativistic expression allowing the Lagrangian representation with respect to the charged particle $\xi$ rest reference frame $\mathcal{K}_{r}\left(\tau, q-q_{f}\right)$, connected with the reference frame $\mathcal{K}(t, q)$ by means of the classical relativistic proper time relationship:

$$
d t=d \tau\left(1+\left|\dot{q}-\dot{q}_{f}\right|^{2}\right)^{1 / 2},
$$

where $\tau \in \mathbb{R}$ is the proper time parameter in the rest reference frame $\mathcal{K}_{r}\left(\tau, q-q_{f}\right)$ and, by definition, the derivative $d / d \tau(\ldots):=(\ldots)$.

Proof Take the following action functional with respect to the charged particle $\xi$ rest reference frame $\mathcal{K}_{r}\left(\tau, q-q_{f}\right)$ :

$$
S^{(\tau)}:=-\int_{t_{1}\left(\tau_{1}\right)}^{t_{2}\left(\tau_{2}\right)} \bar{W} d t=-\int_{\tau_{1}}^{\tau_{2}} \bar{W}\left(1+\left|\dot{q}-\dot{q}_{f}\right|^{2}\right)^{1 / 2} d \tau,
$$

where the proper temporal values $\tau_{1}, \tau_{2} \in \mathbb{R}$ are considered to be fixed in contrast to the temporal parameters $t_{2}\left(\tau_{2}\right), t_{2}\left(\tau_{2}\right) \in \mathbb{R}$ depending, owing to (3.5), on the charged particle $\xi$ trajectory in the phase space. The least action condition

$$
\delta S^{(\tau)}=0, \quad \delta q\left(\tau_{1}\right)=0=\delta q\left(\tau_{2}\right)
$$

applied to (3.6), yields the dynamical equation (3.1), which is equivalent to the relativistic Lorentz type force expression (2.7).

Indeed, compute first the least action variational condition (3.6) with the corresponding Lagrangian function

$$
\mathcal{L}:=-\bar{W}\left(1+\left|\dot{q}-\dot{q}_{f}\right|^{2}\right)^{1 / 2} .
$$

For the generalized particle momentum one finds

$$
\begin{aligned}
\tilde{p} & :=\partial \mathcal{L} / \partial \dot{q}=-\bar{W}\left(\dot{q}-\dot{q}_{f}\right)\left(1+\left|\dot{q}-\dot{q}_{f}\right|^{2}\right)^{-1 / 2} \\
& =-\bar{W} \dot{q}\left(1+\left|\dot{q}-\dot{q}_{f}\right|^{2}\right)^{-1 / 2}+\bar{W} \dot{q}_{f}\left(1+\left|\dot{q}-\dot{q}_{f}\right|^{2}\right)^{-1 / 2} \\
& =m u+\xi A:=p+\xi A
\end{aligned}
$$

and the corresponding dynamical equation with respect the rest reference frame $\mathcal{K}_{r}(\tau, q-$ $q_{f}$ ) is then given as

$$
\frac{d}{d \tau}(p+\xi A)=-\nabla \bar{W}\left(1+\left|\dot{q}-\dot{q}_{f}\right|^{2}\right)^{1 / 2} .
$$

Taking into account that $d \tau=d t\left(1-\left|u-u_{f}\right|^{2}\right)^{1 / 2}$ and $\left(1+\left|\dot{q}-\dot{q}_{f}\right|^{2}\right)^{1 / 2}=(1-\mid u-$ $\left.\left.u_{f}\right|^{2}\right)^{-1 / 2}$, we obtain finally from (3.10) exactly the dynamical system (3.1), finishing the proof. 
3.2 A Moving Charged Point Particle Lagrangian Analysis-A Dual to the Classical Alternative Electrodynamic Model

It is easy to observe that the action functional (3.6) is written taking into account the classical Galilean transformations of the corresponding reference frames. If we now consider this action functional (3.6) for a charged point particle $\xi$, moving with respect the reference system $\mathcal{K}(t, q)$, and take formally into account its interaction with an external magnetic field, generated by the vector potential $A: \mathbb{M}^{4} \rightarrow \mathbb{E}^{3}$, defined on the Minkowski space $\mathbb{M}^{4}:=\mathbb{R} \times \mathbb{R}^{3}$, it can be artificially generalized as

$$
S:=\int_{t_{1}}^{t_{2}}(-\bar{W} d t+q\langle A, d q\rangle)=\int_{\tau_{1}}^{\tau_{2}}\left[-\bar{W}\left(1+\dot{q}^{2}\right)^{1 / 2}+q\langle A, \dot{q}\rangle\right] d \tau,
$$

where we accepted here that $d \tau=d t\left(1-u^{2}\right)^{1 / 2}$ subject to the corresponding rest reference frame $\mathcal{K}(\tau, q)$.

Thus, the corresponding common particle-field momentum looks as follows:

$$
\begin{aligned}
\tilde{p} & :=\partial \mathcal{L} / \partial \dot{q}=-\bar{W} \dot{r}\left(1+|\dot{q}|^{2}\right)^{-1 / 2}+\xi A \\
& =m u+\xi A:=p+\xi A,
\end{aligned}
$$

satisfying the dynamical equation

$$
\begin{aligned}
d \tilde{p} / d \tau & :=\partial \mathcal{L} / \partial q=-\nabla \bar{W}\left(1+|\dot{q}|^{2}\right)^{1 / 2}+\xi \nabla\langle A, \dot{q}\rangle \\
& =-\nabla \bar{W}\left(1-u^{2}\right)^{-1 / 2}+\xi \nabla\langle A, u\rangle\left(1-u^{2}\right)^{-1 / 2},
\end{aligned}
$$

where

$$
\mathcal{L}:=-\bar{W}\left(1+|\dot{q}|^{2}\right)^{1 / 2}+\xi\langle A, \dot{q}\rangle
$$

is the Lagrangian function with respect to the rest reference frame $\mathcal{K}(\tau, q)$. Taking now into account that $\bar{W}:=\xi \varphi$ and $d \tau=d t\left(1-u^{2}\right)^{1 / 2}$, one easily finds from (3.13) that

$$
d \tilde{p} / d t=\xi(-\nabla \varphi-\partial A / \partial t)+\xi \nabla\langle A, u\rangle .
$$

Upon substituting (2.10) into (3.15) and making use of the identity (3.3) we finally obtain from (3.15) the expression for the classical Lorentz force

$$
d p / d t:=\xi E+\xi u \times B,
$$

acting on the moving charged point particle $\xi \in \mathbb{R}$.

The result obtained we formulate as the next proposition.

Proposition 3.3 The classical relativistic Lorentz force (3.16) allows the least action formulation (3.11) with respect to the "rest" reference frame, where Lagrangian function is given by expression (3.14). Nonetheless, its electrodynamics, described by the classical Lorentz force expression (3.16), is not equivalent to the classical relativistic moving point particle electrodynamics, described by means of the Lorentz force expression (3.16), derived from the classical least action functional

$$
S:=\int_{\tau_{1}}^{\tau_{2}} d \tau\left[-m_{0}+\xi\langle A, \dot{q}\rangle-\xi \varphi\left(1+|\dot{q}|^{2}\right)^{1 / 2}\right],
$$


with respect to the rest reference frame $\mathcal{K}(\tau, q)$, where $m_{0} \in \mathbb{R}_{+}$is the Lorentz invariant "rest" mass of a point charged particle.

Making use of the relationships between the reference frames $\mathcal{K}(t, q)$ and $\mathcal{K}_{r}\left(\tau, q-q_{f}\right)$ at case when the external charge particle velocity $u_{f}=0$, we can easily derive the following corollary.

Corollary 3.4 Let the external charge distribution $\xi_{f}$ be in the rest, that is the velocity $u_{f}=0$. Then (3.1) reduces to

$$
\frac{d}{d t}(-\bar{W} u)=-\nabla \bar{W}
$$

allowing the following conservation law:

$$
H_{0}=\bar{W}\left(1-u^{2}\right)^{1 / 2}=-\left(\bar{W}^{2}-p^{2}\right)^{1 / 2} .
$$

Moreover, (3.18) is Hamiltonian with respect to the canonical Poisson structure (1.5), Hamiltonian function (3.19) and the rest reference frame $\mathcal{K}_{r}(\tau, q)$ :

$$
\left.\left.\begin{array}{l}
d q / d \tau:=\partial H_{0} / \partial p=p\left(\bar{W}^{2}-p^{2}\right)^{-1 / 2} \\
d p / d \tau:=-\partial H_{0} / \partial q=-\bar{W}\left(\bar{W}^{2}-p^{2}\right)^{-1 / 2} \nabla \bar{W}
\end{array}\right\} \Rightarrow \begin{array}{l}
d q / d t=-p \bar{W}^{-1} \\
d p / d t=-\nabla \bar{W}
\end{array}\right\} .
$$

In addition, if the rest particle mass $m_{0}:=-\left.H_{0}\right|_{u=0}$, the "inertial" particle mass quantity $m \in \mathbb{R}$ obtains the well known classical relativistic form

$$
m=-W=m_{0}\left(1-u^{2}\right)^{-1 / 2}
$$

depending on the particle velocity $u \in \mathbb{R}^{3}$.

As for the general case of (3.1) results analogous to the above results hold, as described in detail in [15-18]. We need only mention that the Hamiltonian structure of the general equation (3.1) results naturally from its least action representation (3.7) with respect to the rest reference frame $\mathcal{K}(\tau, q)$.

\section{Conclusion}

We have demonstrated the complete legacy of the Feynman's approach to the Lorentz force based derivation of the Maxwell electromagnetic field equations. Moreover, we have succeeded in finding the exact relationship between Feynman's approach and the vacuum field approach devised in $[17,18]$. Thus, the results obtained confirm the deep physical backgrounds lying in the vacuum field theory approach, based on which one can simultaneously describe the physical phenomena both in electromagnetic and gravity terms. The latter is physically related with the particle 'inertial' mass expression (2.18), naturally following both from Feynman's approach to the Lorentz type force derivation and from the vacuum field approach.

Acknowledgements The authors are cordially appreciated to Prof. Z. Peradzynski for useful discussions of the work. The last but not least thanks go to Referees for the instrumental remarks and comments which strongly helped to amend the exposition of main results. 
Open Access This article is distributed under the terms of the Creative Commons Attribution Noncommercial License which permits any noncommercial use, distribution, and reproduction in any medium, provided the original author(s) and source are credited.

\section{References}

1. Dyson, F.J.: Feynman's proof of the Maxwell equations. Am. J. Phys. 58, 209-211 (1990)

2. Dyson, F.J.: Feynman at Cornell. Phys. Today 42(2), 32-38 (1989)

3. Lee, C.R.: The Feynman-Dyson proof of the gauge field equations. Phys. Lett. A 148, 146-148 (1990)

4. Silagadze, Z.K.: Feynman's derivation of Maxwell equations and extra dimensions (2002). arXiv:hep$\mathrm{ph} / 0106235$

5. Brehme, R.W.: Comment on Feynman's proof of the Maxwell equations by F.J. Dyson [Am. J. Phys. 58, 209-211 (1990)]. Am. J. Phys. 59, 85-86 (1991)

6. Dombey, N.: Comment on Feynman's proof of the Maxwell equations by F.J. Dyson [Am. J. Phys. 58, 209-211 (1990)]. Am. J. Phys. 59, 85 (1991)

7. Anderson, J.L.: Comment on Feynman's proof of the Maxwell equations by F.J. Dyson [Am. J. Phys. 58, 209-211 (1990)]. Am. J. Phys. 59, 86 (1991)

8. Farquar, I.E.: Comment on Feynman's proof of the Maxwell equations by F.J. Dyson [Am. J. Phys. 58, 209-211 (1990)]. Am. J. Phys. 59, 87 (1991)

9. Hughes, R.J.: On Feynman's proof of the Maxwell equations. Am. J. Phys. 60, 301-306 (1992)

10. Tanimura, S.: Relativistic generalization and extension to the nonabelian gauge theory of Feynman's proof of the Maxwell equations. Ann. Phys. 220, 229-247 (1992)

11. Vaidya, A., Farina, C.: Can Galilean mechanics and full Maxwell equations coexist peacefully? Phys. Lett. A 153, 265-267 (1991)

12. Abraham, R., Marsden, J.: Foundations of Mechanics, 2nd edn. Benjamin Cummings, Redwood City (1978)

13. Kupershmidt, B.A.: Infinite-dimensional analogs of the minimal coupling principle and of the Poincare lemma for differential two-forms. Differ. Geom. Appl. 2, 275-293 (1992)

14. Bogolubov N.N. Jr., Prykarpatsky, A.K., Taneri, U., Prykarpatsky, Y.A.: The electromagnetic Lorentz condition problem and symplectic properties of Maxwell- and Yang-Mills-type dynamical systems. J. Phys. A, Math. Theor. 42, 165401 (2009) (16pp)

15. Bogolubov N.N. Jr., Prykarpatsky, A.K.: The analysis of Lagrangian and Hamiltonian properties of the classical relativistic electrodynamics models and their quantization. Found. Phys. 40, 469-493 (2010)

16. Prykarpatsky, A.K., Bogolubov N.N. Jr., Taneri, U.: The vacuum structure, special relativity and quantum mechanics revisited: a field theory no-geometry approach. Theor. Math. Phys. 160(2), 1079-1095 (2009). arXiv:0807.3691 v.8 [gr-gc] 24.08.2008

17. Prykarpatsky, A.K., Bogolubov N.N. Jr., Taneri, U.: The field structure of vacuum, Maxwell equations and relativity theory aspects. Preprint ICTP, Trieste, IC/2008/051. http://publications.ictp.it

18. Prykarpatsky, A.K., Bogolubov N.N. Jr., Taneri, U.: The relativistic electrodynamics least action principles revisited: new charged point particle and hadronic string models analysis. Int. J. Theor. Phys. 49, 798-820 (2010)

19. Martins, A.A., Pinheiro, M.J.: On the electromagnetic origin of inertia and inertia mass. Int. J. Theor. Phys. 47, 2706-2715 (2008)

20. Bogolubov, N.N., Prykarpatsky, A.K.: The Lagrangian and Hamiltonian formalisms for the classical relativistic electrodynamical models revisited. arXiv:0810.4254 v1 [gr-qc], 23 Oct. 2008

21. Prykarpatsky, Ya.A., Samoylenko, A.M., Prykarpatsky, A.K.: The geometric properties of reduced symplectic spaces with symmetry, their relationship with structures on associated principle fiber bundles and some applications. Part 1. Opuscula Mathematica 25(2), 287-298 (2005)

22. Prykarpatsky, Ya.A.: Canonical reduction on cotangent symplectic manifolds with group action and on associated principal bundles with connections. J. Nonlinear Oscill. 9(1), 96-106 (2006)

23. Repchenko, O.: Field Physics. Moscow, Galeria Publ. (2005) (in Russian)

24. Hojman, S., Shepley, L.C.: No Lagrangian? No quantization! J. Math. Phys. 32, 142-146 (1991) 